\title{
Fuzzy and Utility Based Network Selection for Heterogeneous Networks in High-Speed Railway
}

\author{
Xiaoyun Yan, Ping Dong, Tao Zheng, and Hongke Zhang \\ Beijing Jiaotong University, Beijing, China \\ Correspondence should be addressed to Ping Dong; pdong@bjtu.edu.cn
}

Received 25 May 2017; Accepted 18 September 2017; Published 30 October 2017

Academic Editor: Yin Xuefeng

Copyright ( 2017 Xiaoyun Yan et al. This is an open access article distributed under the Creative Commons Attribution License, which permits unrestricted use, distribution, and reproduction in any medium, provided the original work is properly cited.

\begin{abstract}
Due to the complexity and fluctuation of the wireless network statuses in the high-speed railway scenario, the existing works of the network selection problem in heterogeneous wireless networks face two major challenges, that is, the imprecise statuses and mobility. In this paper, we propose FSNS, a novel dynamic imprecise-aware network selection approach to solve the problems. The imprecise statuses are inferred by fuzzy rules and the status-awareness feature of the status monitor module enables a dynamic network selection. Through the status monitor and fuzzy processing modules, FSNS is formulated as utility functions for meeting quality-of-service (QoS) requirements. What is more, we carry out plenty of simulation experiments and compare FSNS approach with an enhanced TOPSIS method and fuzzy MADM (FMADM) scheme through simulation experiments of two types of services. The results indicate that FSNS outperforms both TOPSIS and FMADM for a good performance improvement and a preferable decision to keep relative stability and reduce abnormal selections. The conclusion of experimental results has some extent pragmatic value since the network statuses of the simulation are complex and fluctuate by real-world data from high-speed railways.
\end{abstract}

\section{Introduction}

Heterogeneous wireless networks (HWNs) generally integrate multiple wireless access technologies to provide ubiquitous network services [1], such as small cells for 3G, 4G cellular networks [2], Wi-Fi, and LTE networks [3]. As one of the pivotal issues in HWNs, the network selection problem has attracted great attention. It is responsible for selecting the best one from multiple coexistent wireless networks that have heterogeneous characteristics, to conform to the requirements of services. This selection principle is called always best connected $(\mathrm{ABC})$ [4]. Due to the $\mathrm{ABC}$ principle of network selection, varieties of network selection methods using mathematical models have been proposed. Wang and Kuo illustrated several classical mathematical models for $\mathrm{ABC}$ network selection in [5], including utility theory, multiple attribute decision-making (MADM), fuzzy logic, game theory, combinatorial optimization, and Markov chain. These methods have their own respective characteristics and advantages. However, it is difficult to select the accurate network since the wireless network statuses are complex and fluctuate in the high-speed railway (HSR) scenario.
An example of the HSR scenario in HWNs is shown in Figure 1. A high-speed train drives through an area which coexists with one $\mathrm{Wi}-\mathrm{Fi}$, one $3 \mathrm{G}$, and two LTE wireless networks. The users with different $\mathrm{QoS}$ requirements on the train may firstly select $3 \mathrm{G}$ as the $\mathrm{ABC}$ choice; after a period of time, they may select LTE2 as the ABC choice since the vehicle position of the train is changing as time goes by. Hence, we can see that the network selection problem is dynamic and it depends on the real-time network statuses in such environment.

Whereas high-speed mobile communications have some differences from public land mobile communications [6], there are two major challenges for the network selection problem which are urgent to solve. One is the high-speed mobility during network selection procedure, and the other is the imprecision of network statuses for making the selection decision. Firstly, some proposed network selection mechanisms, such as MADM [7], game theory [8], and combinatorial optimization [9], are insufficient to solve these two problems and some improvement should be taken. Secondly, several approaches supporting dynamic network selection, such as Markov chain [10] and kernel learning based utility 


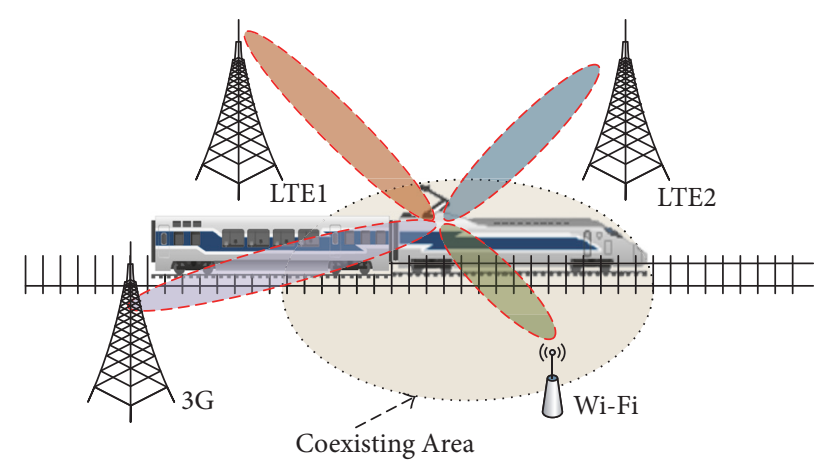

FIGURE 1: An example of high-speed train driving through heterogeneous wireless networks.

method [11], still rely on high precise information. An abnormal selection result might occur by the reason of the imprecision of network statuses. Thirdly, some proposals utilizing fuzzy logic [12] or probability logic [13] to handle the imprecision are inapplicable for high-speed mobility scenarios, since they are designed for either static scenes or a low-I medium-speed mobile environment.

To address the imprecision and dynamic adaptability of network statuses in the HSR scenario and meet different QoS requirements, we propose a novel dynamic impreciseaware network selection approach. The proposed approach is named FSNS which derives from fuzzy and satisfaction based network selection. FSNS jointly considers the imprecision and the dynamics of network statuses with user requirements and has three main advantages. Firstly, FSNS implements a network status monitor module. The network statusawareness feature of this module enables a dynamic network selection. Secondly, it uses the well-known theoretical model of fuzzy logic. Leveraging the inference system of fuzzy logic, imprecise attributes of network statuses and their vague weights which represent service preference are primarily inferred by expert knowledge and service requirements before the decision-making. Thirdly, utility functions are used to conform to QoS requirements. The final decision of network selection is based on the overall satisfaction which is calculated by several utility functions and preference weights. The pseudocode of FSNS algorithm is designed to select the best network with maximal satisfaction. What is more, we carry out plenty of simulation experiments and compare FSNS approach with an enhanced TOPSIS method and FMADM scheme through simulation experiments of two types of services. The evaluation results indicate that FSNS outperforms both TOPSIS and FMADM for a good performance improvement and a preferable decision to maintain relative stability and reduce abnormal selections.

In particular, our contributions are summarized below:

(1) We propose a novel dynamic imprecise-aware network selection approach for the HSR scenario. Compared with the existing methods, FSNS is proposed not only for the imprecision related to the decisionmaking selection but also for the dynamical adaptation to high-speed mobility.
(2) We carry out plenty of simulation experiments by using the real-world data and validate the feasibility and performance of FSNS mechanism. The experiment configuration imitates the high-speed mobile environment by real-world data from high-speed railways, which has some extent practical value.

The remainder of the paper is structured as follows. In Section 2, we review a number of related works and background. In Section 3, we briefly outline fuzzy logic and utility theory as preliminaries. In Section 4, we detail the model and algorithm of FSNS. In Section 5, we present experimental results and discussion of performance improvement compared with enhanced TOPSIS and FMADM. Finally, we conclude the paper and discusses some future directions in Section 6.

\section{Related Work}

There are two major challenges for the network selection problem in high-speed mobility scenarios, namely, the highspeed mobility and the imprecision of network statuses. In general, the network statuses are relatively stable and the measurements of network attributes of selection decision are reliable. Nevertheless, the wireless network statuses are complex and fluctuate in high-speed mobility scenarios. For example, Ai et al. [6] presented the differences between high-speed mobile communications and public land mobile communications. Xiao et al. [14] and Li et al. [15] pointed out that the reasons of the fluctuation of performance metrics are the high-speed mobility and handoff.

2.1. Dynamic-Aware Network Selection Solutions. Since the mobility is a key feature in wireless and mobile networks, several dynamic network selection solutions were proposed in mobile networks [11, 16, 17].

Berg et al. [11] proposed a dynamic network selection method in mobile environments, which took advantage of online kernel learning algorithm and multiattribute utility theory. The online learning was designed to adapt to nonstationary utilities and mobile environments. However, imperfect and imprecise information learning might incur a wrong selection. In addition, the learning speed has a negative effect on the selection results in high-speed mobility scenarios.

Markov decision process (MDP) was employed in vertical handoff decision for HWNs [16]. It made a selection decision based on the status transition probability at each epoch, which attempted to obtain the maximal reward per connection. The decision-making speed of MDP based approach has been improved compared with [11], but it still relies on a high precision of decision-making information.

To reduce the abnormal selection in mobile environments, an enhanced TOPSIS with fast decision speed was proposed [17]. It decreased the normalization impact of changes of network statuses on the rank order with several improved normalized methods. Nevertheless, it still depends on the accurate information of multiple attributes although the enhanced TOPSIS is able to adapt to dynamic network statuses and has a fast decision-making speed. 
2.2. Imprecise-Aware Network Selection Solutions. The imprecision or uncertainty of decision-making information is another key issue. To deal with imprecise information, some schemes were proposed to leverage fuzzy logic and probability theory in [18-20].

Fuzzy logic based scheme was presented to select the appropriate network for applications in wireless vehicular network [18]. Distinct from previously mentioned selection methods, it took received signal strength indicator (RSSI), network density, vehicle speed, and service cost into consideration to infer a matching network with the corresponding application. However, the fuzzy inference rules with three levels had a coarse granularity. Moreover, it focuses on urban road vehicles while the velocity is not exceeding $100 \mathrm{~km} / \mathrm{h}$ and lacks the adaptation to network status change in high-speed mobility.

A belief function theory (BFT) approach [19] was proposed to cope with conflicting beliefs due to the uncertainty of network statuses information. Multiple attributes and their weights associated with a specific application were considered to rank the available network. However, it is just numerical analysis in several static scenarios rather than realistic dynamic experiments and is not adaptive to highspeed mobility scenarios.

FMADM was proposed to handle the imprecision related to decision-making [20]. It combines fuzzy logic and multiple attribute decision-making algorithm to make an access decision. However, it is just the static scene analysis rather than realistic dynamic results which may be not adaptive for highspeed mobility scenarios.

2.3. Design Consideration. Combining the analysis of existing works mentioned above with the high-speed railway environment, the design considerations of selecting an appropriate candidate network to satisfy users' requirements are listed below.

(i) Dynamics: network statuses and performance metrics involved with the network selection decision are dynamically changing. Thus, we need to design a dynamical network selection mechanism that could dynamically perceive the status information of candidate networks and select the best one of them according to the updated information.

(ii) Imprecision: during the network selection process, the heterogeneity of different wireless networks may lead to the imprecise selection criteria, and service requirements for network attributes are often vague. In consequence, the design mechanism needs to cope with the imprecision of decision-making information.

(iii) Complexity: the feature of high-speed mobility requires a fast decision speed. Hence, the time complexity of theoretic algorithms used in the design mechanism cannot be too high. For example, the negotiation process of game theory and the kernel learning process take too much time that should not be considered.

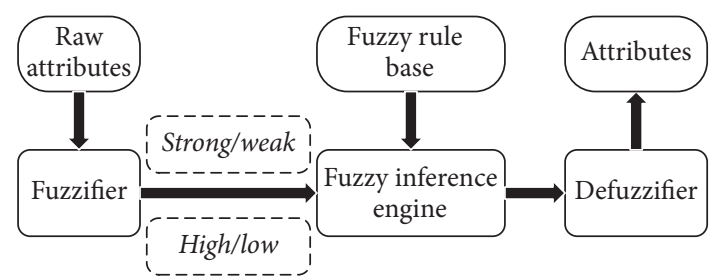

Figure 2: A basic framework of the fuzzy processing.

\section{Preliminaries}

In this section, we briefly outline fuzzy logic and utility theory and describe some model settings that are utilized to formulate the network selection problem in high-speed mobility scenarios.

3.1. Fuzzy Logic. Fuzzy logic introduces the conception of the degree in the authorized verification of a condition which is beneficial to take into consideration inaccuracies and uncertainties [21]. Hence, fuzzy logic is very suitable to cope with the imprecise information by an inference system and series of fuzzy rules during the network selection procedure [12].

To solve the imprecision of the network selection problem, fuzzy logic directly infers the ABC network (called direct type) or only infers the status of an imprecise attribute for other decision-making models (called indirect type). The two types are the same in terms of the imprecise information processing; we can only take one of them as an example. Due to the combination of fuzzy logic and utility theory for the network selection problem in this paper, we take the indirect type as the example. A basic framework of the indirect type of the fuzzy processing is shown in Figure 2 and some concepts of the fuzzy processing are explained as follows.

Raw Attributes. Raw attributes are the input variables which are imprecise and would be used to make a decision during the network selection problem. In this paper, bandwidth (BW), received signal strength (RSS), and signal to noise ratio (SNR, or signal to interference and noise ratio (SINR)) are imprecise attributes. Since different wireless communication systems have different signal strength standard, such as SINR, reference signal received power (RSRP) for LTE and $\mathrm{SNR}$, received signal strength indicator (RSSI) for Wi-Fi, the parameters of wireless signal quality are imprecise. In addition, an inaccurate selection result may be obtained as a result of the vague bandwidth requirement of application service.

Fuzzifier. It maps a series of crisp numbers of imprecise attributes (i.e., raw attributes) to a fuzzy set of linguistic descriptions according to expert knowledge and user involvement [22]. The crisp numbers are the measurement of raw attributes and the fuzzy set of linguistic descriptions expresses an acceptable level or relative degree. The frequently used sets consist of two levels (e.g., "high/low" and "strong/weak") in Figure 2. The granularity of status division of bandwidth can be increased to improve the accuracy of decision-making, 
but the computational overhead of the terminal device will also increase. From the view of the speed and complexity of making the decision, five levels (i.e., "very-low," "low," "medium," "high," and "very-high") are introduced in this paper.

Fuzzy Rule Base. It covers a series of fuzzy rules to infer the scope of output variables by these linguistic descriptions. A basic form of fuzzy rules is if-then.

Fuzzy Inference Engine. It uses the fuzzy rule base to infer the linguistic status of output variables. It infers the corresponding statuses of output variables by the input variables and ifthen rules.

Membership Function. It is the mapping function during the defuzzifier process. Chen and Hwang [22] proposed several scales of membership functions to convert fuzzy variables into crisp numbers.

Defuzzifier. It is an inverse process of fuzzifier that maps the linguistic descriptions to crisp numbers valued in $[0,1]$. The mapping relationship is called membership function. According to the conversion of the defuzzifier procedure [22], the five levels are converted into triangular fuzzy numbers $\{0.091,0.283,0.5,0.717,0.909\}$.

Attribute. Attributes are the output crisp variables which are used to make a decision during the network selection problem. They are valued in $[0,1]$ standing for the acceptable degrees.

At the beginning of the fuzzy processing, the imprecise attributes are fuzzified to a fuzzy set of linguistic descriptions. Then, the output variables are inferred to the corresponding statuses by using the fuzzy inference engine. Finally, the linguistic statuses of out variables are converted into crisp numbers by membership functions. Since the fuzzy set and fuzzy rules could be set in advance, the fuzzy processing will not take too much time. Therefore, from the point of the speed and complexity of making the decision in high-speed mobility, fuzzy logic is suitable to deal with the imprecision.

3.2. Utility Theory. Utility theory utilizes the satisfaction of a service provider to the decision-maker for making the decision [23] and the candidate with the best satisfaction always is selected. The satisfaction is always calculated by several utility functions involved with multiple requirements and the preference of the decision-maker. Consequently, utility theory is suitable for dealing with various QoS requirements during the network selection problem.

In this paper, the attributes of QoS requirements used to calculate the satisfaction cover the network performance metrics and wireless link quality parameters. The set of these attributes is assumed as $\mathbf{X}=\{\mathrm{BW}$, delay, jitter, PLR, RSS, SNR\}, while jitter means delay jitter and PLR means packet loss ratio. The utility function of an attribute generally is different from others. For example, the upward attribute and downward attribute have the adverse impact on making the decision. The higher value of the upward attribute

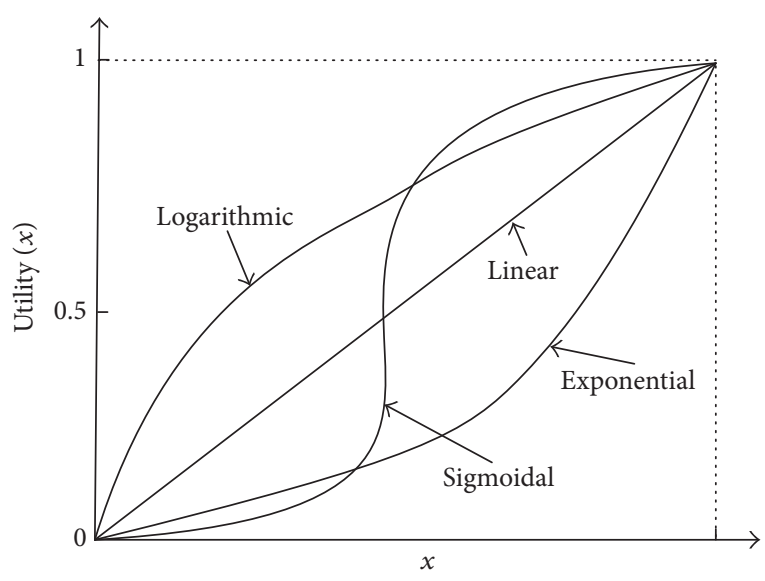

FIgURE 3: Examples of utility functions.

corresponds to a higher value of utility and the lower value of the downward attribute corresponds to a higher value of utility [5]. We notice that the upward attributes in $\mathbf{X}$ include BW, RSS, and SNR, and the downward attributes include delay, jitter, and packet loss ratio. In addition, the utility can be formulated into several functions. For the upward attribute, some utility functions are shown in Figure 3, including logarithmic, exponential, sigmoidal, and linear functions. Since the selection decision is influenced by multiple attributes, the overall satisfaction is calculated by a combination of utilities of multiple attributes. The combinational function integrates not only diversified utility functions of all the attributes, but also their weights involved with service preference.

\section{FSNS Mechanism}

In this section, the details of the proposed fuzzy and utility based network selection approach are presented. We first model the fuzzy processing for the imprecision of network statuses and the vagueness of service preference during the selection procedure. Then we utilize the utility functions of multiple attributes which contain the fuzzified and raw precise attributes to compute the satisfactions of the candidate networks to services. Finally, we present FSNS algorithm using pseudocode.

In our proposed model, FSNS algorithm integrates two classical theories to solve the network selection problem in the HSR scenario. Different from the fuzzy processing in Figure 2, we use fuzzy logic only to cope with the imprecise information during the decision-making procedure not to rank the available networks. For final decision-making, utility theory is utilized for satisfying users' QoS requirements. Abbreviations section displays the parameters used in the model with their descriptions.

4.1. Fuzzy Processing. Taking into consideration imprecise information of network statuses in high-speed mobile environments and vague weights of service preference, the fuzzy logic and inference system are required before the decisionmaking. 
TABLE 1: The fuzzy set of imprecise attributes.

\begin{tabular}{|c|c|c|c|c|c|}
\hline & Very-low & Low & Medium & High & Very-high \\
\hline $\operatorname{RSRP}(\mathrm{dBm})$ & $(-\infty,-115]$ & $(-115,-105]$ & $(-105,-95]$ & $(-95,-85]$ & $(-85,0)$ \\
\hline $\operatorname{SINR}(\mathrm{dB})$ & $(-\infty, 3]$ & $(3,10]$ & $(10,15]$ & $(15,25]$ & $(25, \infty)$ \\
\hline RSSI $(\mathrm{dBm})$ & $(-\infty,-110]$ & $(-110,-101]$ & $(-101,-90]$ & $(-90,-75]$ & $(-75,0)$ \\
\hline Bandwidth (Mbps) & {$[0,0.1]$} & $(0.1,1]$ & $(1,5]$ & $(5,15]$ & $(15,50]$ \\
\hline
\end{tabular}

In this paper, bandwidth, RSSI/RSRP, and SNR/SINR first need to be inferred to the appropriate levels through the fuzzy processing as shown in Figure 2, since they are regarded as imprecise attributes in the network selection model. The other three attributes delay, delay jitter (or jitter for short), and packet loss ratio are directly used in the satisfaction calculation for the final decision-making. Note that the measured bandwidth is the available bandwidth (ABW) in fact; ABW stands for bandwidth without extra explanation in the rest of this paper.

For ease of description, we will use the fuzzy set with five kinds of acceptable levels during the fuzzifier process in Table 1, which are set by expert knowledge and service requirements. RSRP and SINR for LTE and RSSI and SNR for other wireless networks are fuzzified into linguistic descriptions according to the widely accepted conclusion of previous research [24]. The $-\infty$ and $\infty$ symbols in Table 1 stand for the infinite lower and upper bound, respectively, while a finite value will replace them according to the situation in practice. The fuzzy set of BW is based on the QoS requirements of application services [25]. Besides $50 \mathrm{Mbps}$ is the maximum uplink bandwidth of LTE, and $100 \mathrm{Mbps}$ is the downlink bandwidth. Since there are over 200 kinds of highspeed rail-related services (e.g., train control signals, safety monitoring video, and remote fault diagnosis) [6] that need to be transmitted to the ground server, we mainly focus on the upload data from the mobile terminal aboard in this paper.

Similarly, fuzzy logic also is used to determine the weights of multiple attributes considering service preference. Linguistic variables of weights standing for users' preference are converted into crisp numbers using the conversion scale mentioned in [22]. For example, the preference weights of attribute set $\mathbf{X}=\{\mathrm{BW}$, delay, jitter, PLR, RSS, SNR $\}$ for video transmission service are $w f_{v}=\{$ "high", "very-high", "high", "medium", "medium", "medium"\}, corresponding to a crisp weight $w c_{v}=\{0.717,0.909,0.717,0.5,0.5,0.5\}$. The preference weights for file uploading service are $w f_{f}$ $=$ " "medium", "low", "medium", "very-high", "medium", "medium" $\}$, corresponding to a crisp weight $w c_{f}=\{0.5,0.283$, $0.5,0.909,0.5,0.5\}$. Since the weights should be normalized which means $\sum_{i} w_{i}=1$, the crisp weights are normalized as $w_{v}=\{0.1865,0.237,0.1865,0.13,0.13,0.13\}$ and $w_{f}=\{0.1565$, $0.089,0.1565,0.285,0.1565,0.1565\}$ by the following formula.

$$
w(i)=\frac{w c(i)}{\sum_{i} w c(i)} .
$$

The attributes and weights handled by the fuzzy processing are further used to calculate the satisfaction of a network to a service in the next subsection.
TABLE 2: QoS requirements of services.

\begin{tabular}{lcc}
\hline & Video & File uploading \\
\hline Bandwidth (kbps) & {$[1000,6000]$} & {$[50,500]$} \\
Delay $(\mathrm{ms})$ & {$[0,25]$} & {$[100,1000]$} \\
Jitter (ms) & {$[0,25]$} & {$[0,100]$} \\
Packet loss ratio & {$[0,0.01]$} & {$[0,0]$} \\
\hline
\end{tabular}

4.2. Satisfaction Calculation. We now describe the satisfaction calculation by utility functions with the consideration of QoS requirements of services. Moreover, the different services have different preferences for the same network. Therefore, the preference weights of multiple attributes are considered.

As [25] analyzes, the QoS requirements of video transmission and file uploading services are exhibited in Table 2, including bandwidth, delay, jitter, and packet loss ratio. Besides, a mobile terminal (MT) generally has thresholds of signal-related attributes to ensure the normal reception of data. We suppose $\theta^{k}$ denotes the threshold of RSS or SNR/ SINR. The weights of all these attributes for two services are expressed as $w_{v}$ and $w_{f}$, respectively.

We use the utility function to calculate the satisfaction of a candidate network to a service. We assume the service set as $\mathbf{S}=\left\{s_{1}, s_{2}, \ldots\right\}$, which is served by the candidate network set $\mathbf{C N}=\left\{\mathrm{CN}_{1}, \mathrm{CN}_{2}, \ldots,\right\}$. Here, two types of satisfaction calculation formulations (i.e., utility functions) are required: (1) interval type; the value of attribute $k$ is limited in a certain interval, that is, $\left[l_{j}^{k}, u_{j}^{k}\right]$; (2) threshold type. The attribute has a threshold value $\theta_{j}^{k}$, to satisfy the minimal requirement. In consideration of upward and downward attributes, the satisfaction of network $\mathrm{CN}_{i}$ to service $s_{j}$ is defined as follows:

$$
\begin{aligned}
& \mathrm{Sat}_{i j}^{k}= \begin{cases}1, & x_{i}^{k}>u_{j}^{k} \\
\frac{x_{i}^{k}-l_{j}^{k}}{u_{j}^{k}-l_{j}^{k}}, & l_{j}^{k} \leq x_{i}^{k} \leq u_{j}^{k} \\
0, & x_{i}^{k}<l_{j}^{k}\end{cases} \\
& \mathrm{Sat}_{i j}^{k}= \begin{cases}1, & x_{i}^{k}<l_{j}^{k} \\
\frac{u_{j}^{k}-x_{i}^{k}}{u_{j}^{k}-l_{j}^{k},} & l_{j}^{k} \leq x_{i}^{k} \leq u_{j}^{k} \\
0, & x_{i}^{k}>u_{j}^{k}\end{cases} \\
& \operatorname{Sat}_{i j}^{k}= \begin{cases}1, & x_{i}^{k} \geq \theta_{j}^{k} \\
0, & x_{i}^{k}<\theta_{j}^{k}\end{cases}
\end{aligned}
$$


Require: $l_{j}^{k}, u_{j}^{k}, \theta_{j}^{k}, w f_{j}^{k}$ of attribute $k$ for service $s_{j}$;

(1) while each period of monitoring do

(2) Import measurements $\mathbf{X}$ of attributes;

(3) for $\mathrm{CN}_{i} \in \mathrm{CN}$ do

(4) for $x_{i}^{k} \in \mathbf{X}$ do

(5) $\quad x f_{i}^{k}=\operatorname{Fuzzy}\left(x_{i}^{k}\right)$;

(6) end for

(7) end for

(8) for $s_{j} \in \mathbf{S}$ do

(9) $\quad w_{j}^{k}=\operatorname{Fuzzy}\left(w f_{j}^{k}\right)$;

(10) end for

(11) for $s_{j} \in \mathbf{S}$ do

(12) for $\mathrm{CN}_{i} \in \mathbf{C N}$ do

(13) Calculate $\mathrm{Sat}_{i j}^{k}$;

(14) $\quad \mathrm{Sat}_{i j} * w_{j} \Rightarrow \mathrm{Sd}_{i j}$;

(15) end for

(16) if $\forall \mathrm{Sd}_{i j} !=0$ then

(17) Select $\mathrm{CN}_{l}$ with best value $\mathrm{Sd}_{i l}$;

(18) else if $\forall S d_{i j}==0$ then

(19) Select $\mathrm{CN}_{l}$ with best value of preferential attribute $k$;

(20) end if

(21) end for

(22) end while

Algorithm 1: FSNS algorithm.

$$
\mathrm{Sat}_{i j}^{k}= \begin{cases}0, & x_{i}^{k}>\theta_{j}^{k} \\ 1, & x_{i}^{k} \leq \theta_{j}^{k},\end{cases}
$$

where $k$ represents a network attribute. Formulas (2) and (3) both belong to the interval type, but they are applied for inverse attributes. Formula (2) is for positively related attributes (i.e., bandwidth) to compute the satisfaction and formula (3) is for negatively related attributes, such as delay, jitter, and packet loss ratio. Formulas (4) and (5) belong to the threshold type and formula (4) is suitable for attributes like RSS and SNR. Given that RSS, SNR, and BW are processed through the fuzzy processing, $u_{j}^{k}, l_{j}^{k}$, and $\theta_{j}^{k}$ can be defined as the fuzzified linguistic statuses or defuzzified crisp numbers. Therefore, we set the interval $\left[l_{v}^{\mathrm{BW}}, u_{v}^{\mathrm{BW}}\right]$ as ["medium", "high"] for video, $\left[l_{f}^{\mathrm{BW}}, u_{f}^{\mathrm{BW}}\right]$ as ["very-low", "low"] for file, and the threshold $\theta_{j}$ of RSS or SNR as "medium" learning from Tables 1 and 2.

For the purpose of computing the overall satisfaction of network $\mathrm{CN}_{i}$ to service $s_{j}$, the weight set $\mathbf{w}_{j}$ of service $j$ is obtained through fuzzy logic discussed in the previous subsection. Then we can obtain the preference satisfaction according to the following formula.

$$
\mathrm{Sd}_{i j}=\sum_{k=1}^{n} \mathrm{Sat}_{i j}^{k} * w_{j}^{k} .
$$

Depending on the ABC principle, the candidate network with the best value of overall satisfaction $\mathrm{Sd}_{i j}$ is selected.

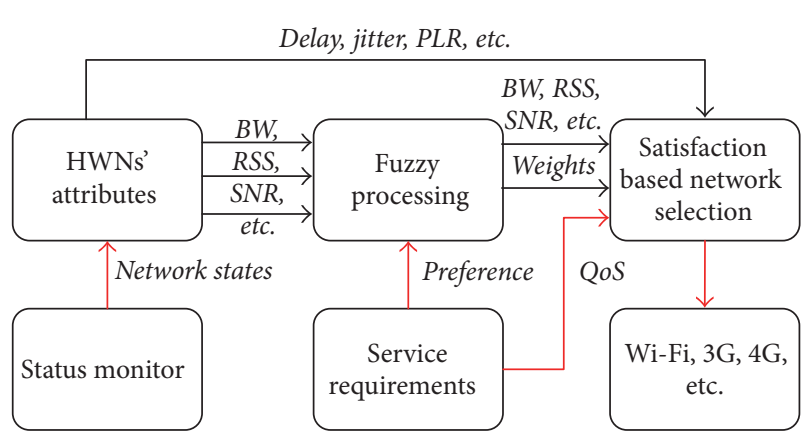

Figure 4: The functional modules of MT.

4.3. FSNS Algorithm. According to the above fuzzy model and satisfaction formulas, we design FSNS algorithm in this subsection. We suppose that FSNS algorithm is deployed in a mobile terminal (MT) equipped with multiple wireless network cards. Consequently, the MT enables us to satisfy multiple services by using several heterogeneous wireless networks. We discuss the functional modules of MT with the help of Figure 4 and present FSNS algorithm in Algorithm 1.

The MT inwardly converges QoS requirements of different services and dynamically monitors network statuses of connected HWNs outwardly. The status monitor module dynamically monitors each available wireless network for MT periodically and transmits network statuses into the next module. The HWNs' attributes module is used to integrate the latest measurements with history's measurements and import the raw attributes into the fuzzy processing module 


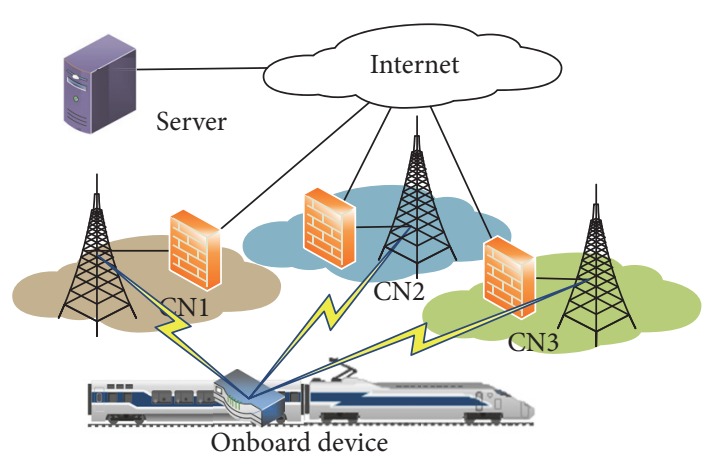

(a) Topological graph

\begin{tabular}{c|c|c|c}
\hline Parameter & $\begin{array}{c}\text { Measurement } \\
\text { period }\end{array}$ & $\begin{array}{c}\text { Network } \\
\text { mode }\end{array}$ & Velocity \\
\hline Value & $3 \mathrm{~s}$ & LTE & $300 \mathrm{~km} / \mathrm{h}$ \\
\hline
\end{tabular}

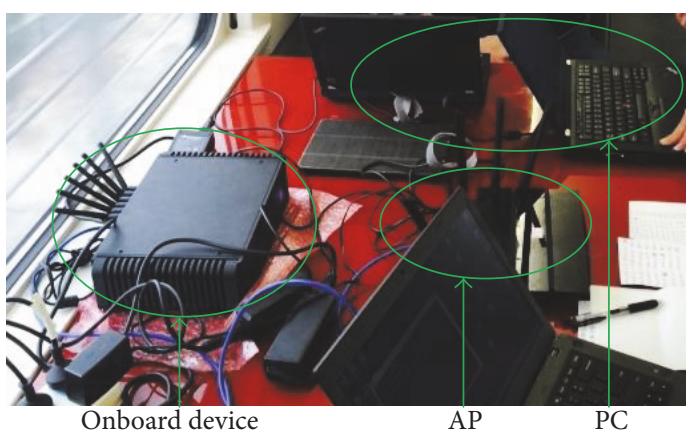

(b) Actual setting and deployment

FIGURE 5: The experimental topological graph and real deployment in high-speed railways.

and the satisfaction based network selection module. The fuzzy processing module converts imprecise attributes into several levels of linguistic descriptions and finally into crisp triangular numbers. Meanwhile, it determines the weights of the attributes for service preference. Finally, the satisfaction based network selection module selects the best network ranking by the overall satisfaction.

With consideration of the above functional modules, the algorithm can be implemented by the pseudocode shown in Algorithm 1. It supposes that the interval and threshold of an attribute for a specific service are known in advance. Lines (1)-(2) periodically import latest and historical measurements to the system. Lines (3)-(10) fuzzify the imprecise attributes of each candidate network $\mathrm{CN}_{i}$ and vague weights in the fuzzy logic module. Lines (11)-(15) calculate service satisfaction for service $s_{j}$ by using formulas (2)-(6). Lines (16)-(17) decide the best network for service $s_{j}$ by the overall satisfaction primarily and lines (18)-(20) suggest that a preferential method is an alternative to avoid an empty selection.

\section{Implementation and Evaluation}

In this section, we describe how we collect the practical data from a real-world high-speed railway. Then we evaluate the performance of FSNS compared with two classical methods TOPSIS and FMADM using these truthful data.

5.1. Data Collection. We have carried out plenty of experiments on real high-speed railways in the recent two years. The lines of actual test covered Beijing-Shanghai, ZhengzhouWuhan, Hefei-Wuhan, and Dalian-Dandong. We collected a large number of real data along the railways. Note that the measurement and collection of multiple attributes including delay, jitter, packet loss ratio, bandwidth, RSRP, and SINR were performed along Beijing-Shanghai high-speed railway with the velocity up to $300 \mathrm{~km} / \mathrm{h}$. In consideration of the wide popularity of LTE communication technologies among users [26] and HSR communication systems [27], we choose three LTE cellular networks as candidate networks, which belong to China Mobile (CM), China Unicom (CU), and China Telecom (CT) operators, respectively.

Figure 5 illustrates the experimental topological graph and an actual experimental scene including the equipment deployment and setting. In the topological graph shown in Figure 5(a), $\mathrm{CN}_{i}(i=1,2,3)$ denotes a cellular network attached to one of three major operators. The onboard device and ground server correspond to the testing client and server $(\mathrm{C} / \mathrm{S})$, respectively. Figure 5(b) displays an actual experimental equipment deployment and setting on a train. The table in Figure 5(b) shows three parameter settings: the measurement period was 3 seconds; the network modes of three candidate cellular networks were LTE; the velocity of HSR was up to $300 \mathrm{~km} / \mathrm{h}$. In the real experimental deployment, the facilities on the train included an onboard device, an access point (AP), and several personal computers (PCs). The equipment on ground included a server. The onboard device was a multichannel router with Intel ATOM D525 quad core $1.8 \mathrm{GHz} \mathrm{CPU}, 1 \mathrm{G}$ memory, and $32 \mathrm{G}$ hard-disk storage. The onboard PCs were Lenovo Thinkpad T series which connected to the onboard device via an AP. The server was a DELL PowerEdge R720 rackmount server with Intel Xeon E5-2609 CPU, 32G memory, and 1TB hard-disk storage. In addition, the onboard device had been installed with the operating system of Ubuntu 12.04 LTS with Linux kernel 3.6.0 [28]. It also had been embedded with data measurement and collection software to realize these main functions. The PCs and server had been deployed with a variety of test software and measurement tools (e.g., iptraf and ftp client/server) for experiments. Since the onboard device was a middleware of $\mathrm{C} / \mathrm{S}$ and was connected with $\mathrm{CNs}$ through base stations, it could capture real-time data packets and channel signals during the $\mathrm{C} / \mathrm{S}$ communication. These captured data finally were calculated and collected as network and channel quality 


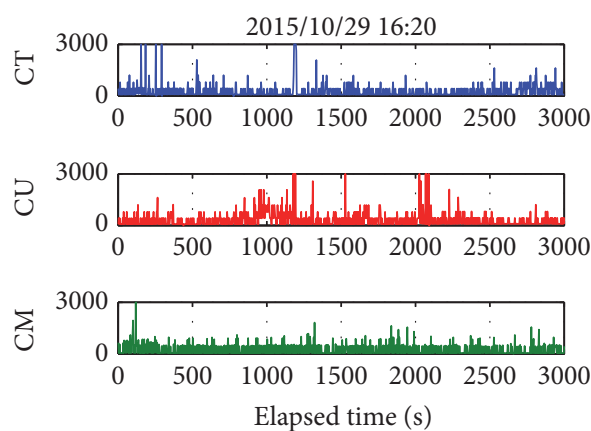

(a) Onboard delay (ms)
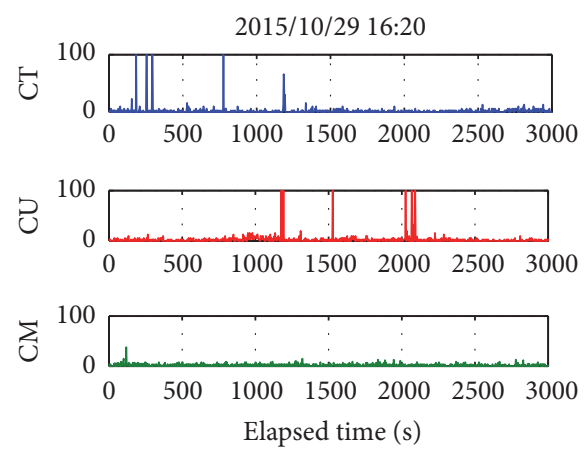

(c) Onboard packet loss ratio (\%)
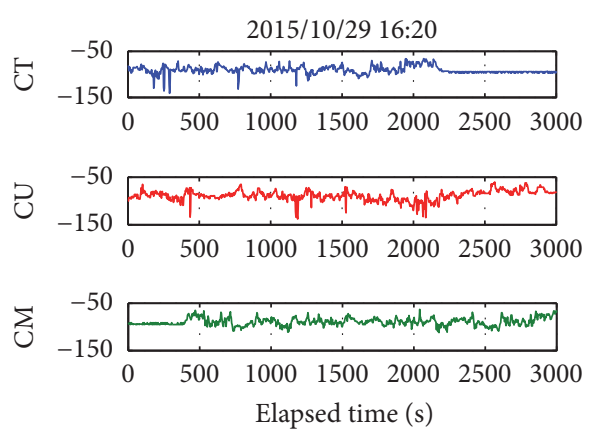

(e) Onboard RSRP $(\mathrm{dBm})$
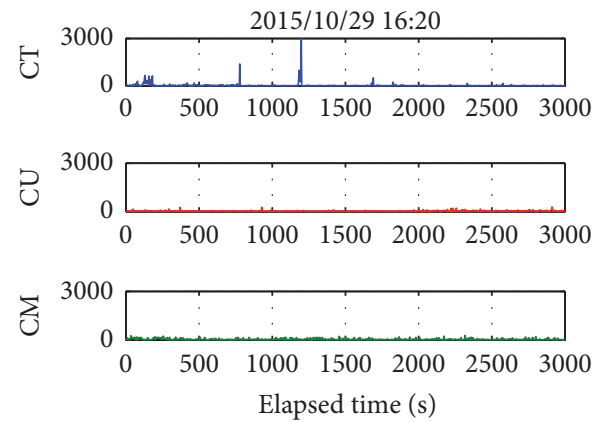

(b) Onboard jitter (ms)
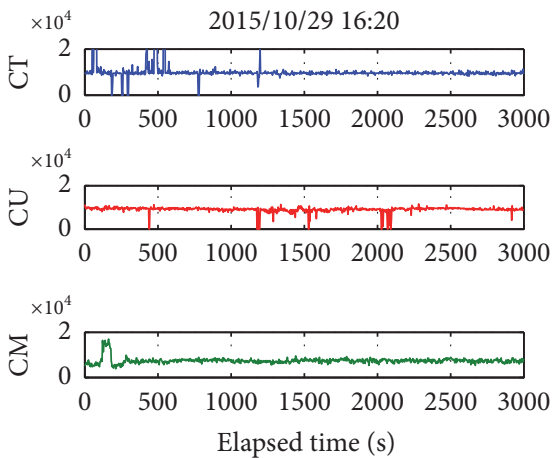

(d) Onboard bandwidth (kbps)
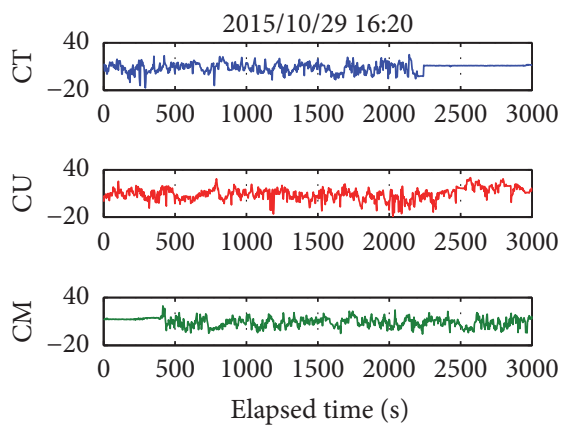

(f) Onboard SINR (dB)

FIGURE 6: Practical measured data collected from Beijing-Shanghai high-speed railway.

parameters by using the data measurement and collection software.

The practical measured data collected from three LTE cellular networks in Beijing-Shanghai high-speed railway are shown in Figures 6(a)-6(f), where $x$-axis is the elapsed time. Similar to the measurement method of round-trip time in [29], 3000 milliseconds (ms) is the upper bound of delay and jitter which is the monitoring period. We show the packet loss ratio as a range of percentage valued $[0,100]$. Therefore, 3000,100 , and 0 indicate the extreme values of delay, packet loss ratio, and bandwidth, respectively. From the figures, we observe that the delay, RSRP, and SINR of these networks fluctuate obviously and all the metrics can be extreme values occasionally. For example, the delays are $3000 \mathrm{~ms}$ at 153 seconds of CT, 1176 seconds of CU, and 117 seconds of CM. The jitter is $3000 \mathrm{~ms}$ at 1197 seconds of CT. The packet loss ratios are $100 \%$ at 252 seconds of CT and 2025 seconds of
CU. The throughputs are 0 at 183 seconds of CT and 438 seconds of CU. These data will be used in the simulation experiments to imitate network statuses in the high-speed mobile environment in the next subsection.

5.2. Performance Evaluation. We carry out experiments that compare FSNS algorithm with an enhanced TOPSIS method and FMADM approach. The simulation experiments generate video transmission service with a random rate valued in $[1000,6000]$ and file uploading service with a random rate valued in $[50,500]$, where the unit is kbps. The practical data collected from real-world high-speed railways are utilized to simulate network statuses of the HSR scenario during the experiments.

5.2.1. Performance Metrics. For convenience, the number of extreme values and statistical analysis (i.e., mean and 

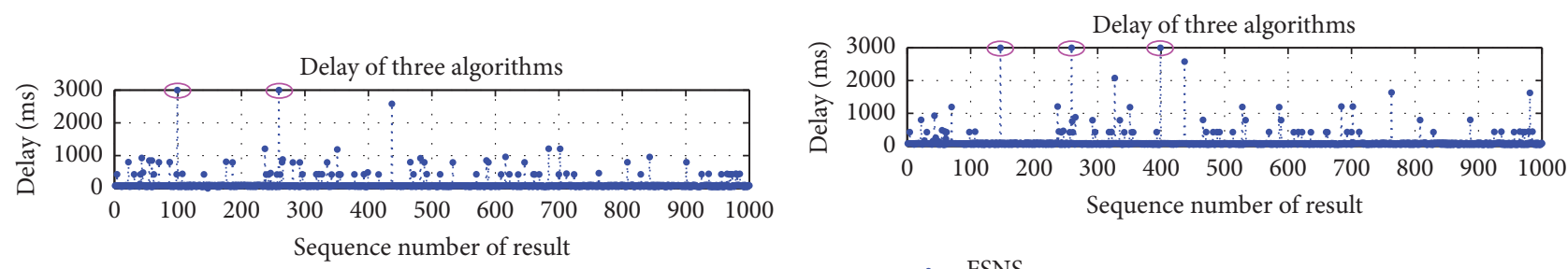

... FSNS

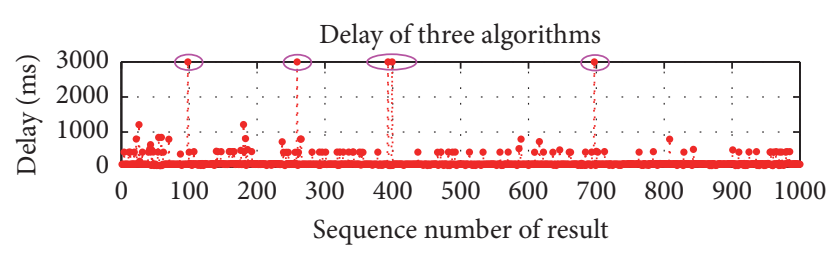

-.. FSNS

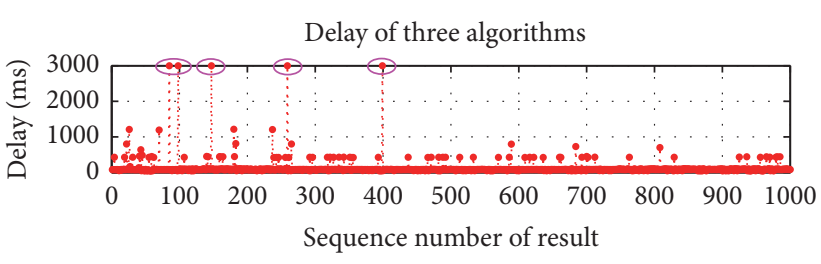

-.. TOPSIS

-.. TOPSIS

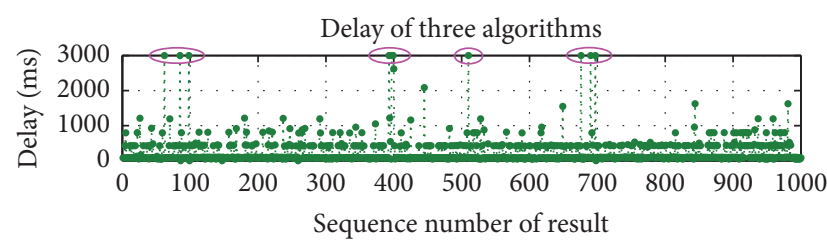

... FMADM

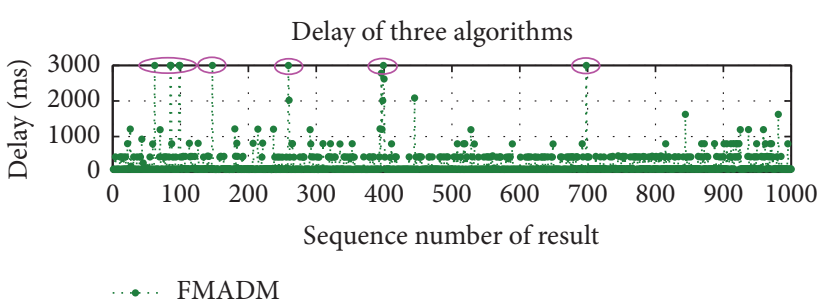

(a) Delay of file service

(b) Delay of video service

FIGURE 7: The delay comparison of three algorithms.

standard deviation) of simulation results are used to evaluate our proposed mechanism.

Number of Extreme Values. These extreme values stand for the poor quality of selected networks. The lower number of extreme values means the better performance of a network selection algorithm. In this paper, 3000, 100, and 0 indicate the extreme values of delay, packet loss ratio, and bandwidth, respectively.

Mean and Standard Deviation. The mean and standard deviation are both statistical parameters. The mean represents the average of a set of data. The standard deviation is the arithmetic square root of the variance, which represents the degree of deviation from the expectation. It suggests the stability performance in terms of delay, jitter, packet loss ratio, and throughput. The formulas of the mean and variance are shown in (7).

$$
\begin{aligned}
\bar{x}^{k} & =\frac{1}{n} \sum_{i=1}^{n} x^{k}(i) \\
\mathrm{sd}^{k} & =\sqrt{\frac{1}{n-1} \sum_{i=1}^{n}\left(x^{k}(i)-\bar{x}^{k}\right)^{2}} .
\end{aligned}
$$

5.2.2. Evaluation Results. The evaluation results of FSNS in comparison with TOPSIS and FMADM by using video and file services are shown in Figures 7-10, consisting of network delay in Figure 7, jitter in Figure 9, packet loss ratio in
Figure 8, and throughput in Figure 10 successively. In (a) and (b) subgraphs of Figures 7-10, the $x$-axis stands for the sequence number of simulation results, where 1000 results correspond to 3000 seconds of elapsed time in the actual environment. The $y$-axis is the value of a performance criterion. The upper bounds of these performance metrics are mainly incurred by the poor quality of selected networks. With the comparison of TOPSIS and FMADM, we analyze the performance improvement of FSNS from the following points.

Firstly, Figure 7 shows the delay performance of FSNS is superior to TOPSIS and FMADM from the following aspects: (1) FSNS decreases the overall delay compared with TOPSIS and FMADM. FSNS only has $7.4 \%$ of results up to $400 \mathrm{~ms}$, in comparison with $8.9 \%$ using TOPSIS and 29\% using FMADM. (2) FSNS reduces the number of extreme delays compared with TOPSIS and FMADM. The number of results reached to $3000 \mathrm{~ms}$ which are marked by pink circles and is reduced by using FSNS. The numbers of file service are 2, 5, and 9 by using FSNS, TOPSIS, and FMADM, respectively. The numbers of video service are 3, 5, and 7, correspondingly. (3) FSNS improves the performance of delay metric from the point of overall differences compared with FMADM.

Secondly, Figure 8 shows the packet loss ratio performance of FSNS has advantage over TOPSIS and FMADM in the following: (1) FSNS decreases the overall packet loss ratio compared with FMADM. FSNS only has $2.8 \%$ of results up to $5 \%$, in comparison with $7.4 \%$ using FMADM. (2) FSNS reduces the number of extreme packet loss ratios compared with TOPSIS and FMADM. The number of results reached 


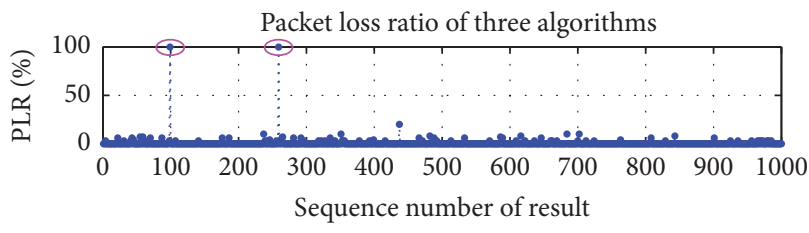

... FSNS

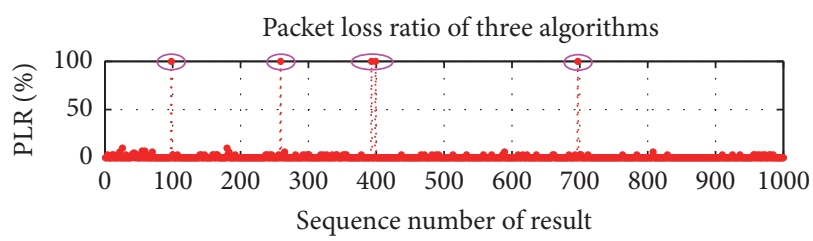

... TOPSIS

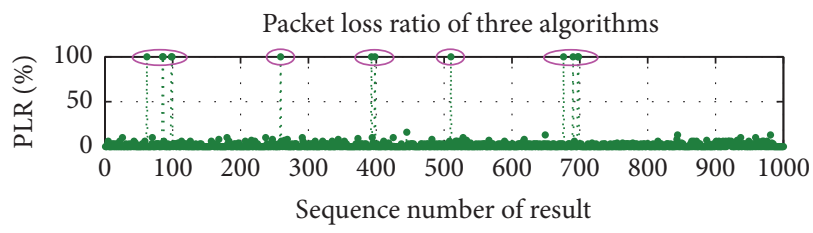

... FMADM

(a) Packet loss ratio of file service

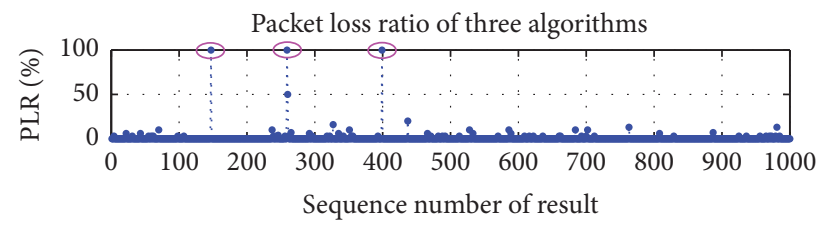

... FSNS

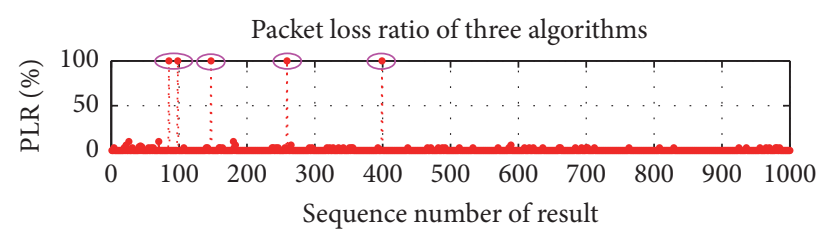

.. TOPSIS

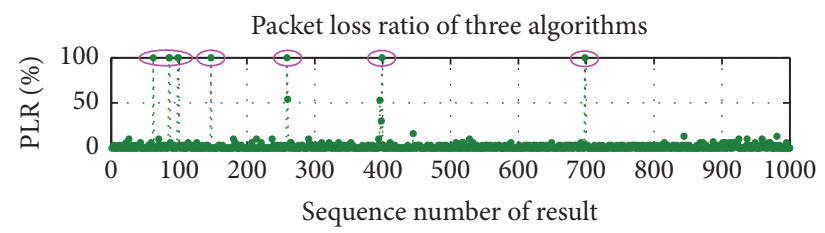

-.. FMADM

(b) Packet loss ratio of video service

Figure 8: The packet loss ratio comparison of three algorithms.
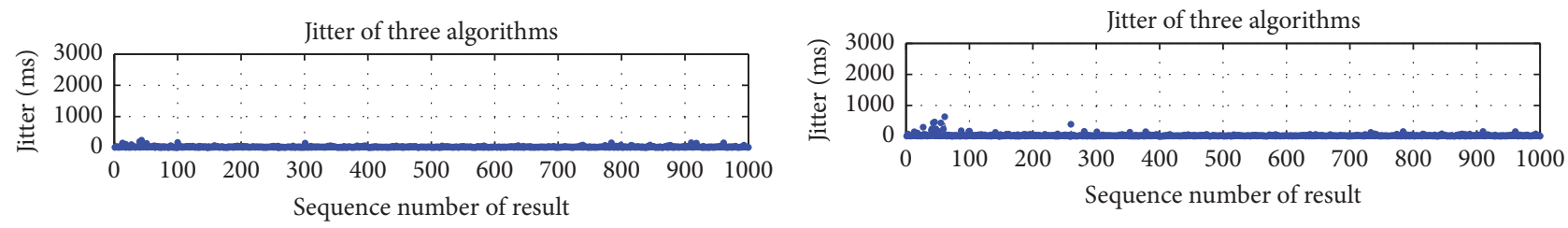

... FSNS

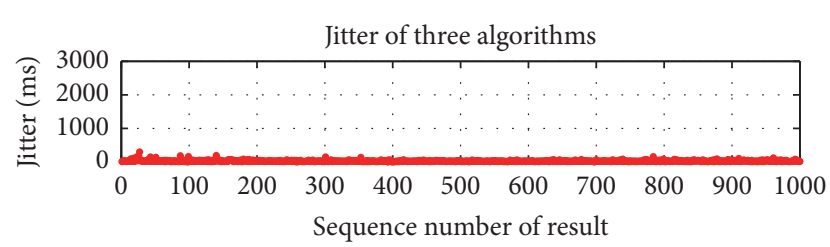

... FSNS

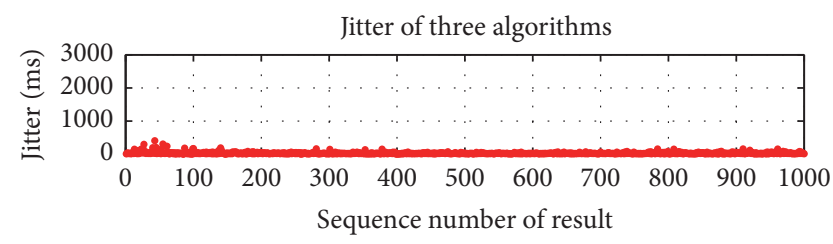

-.. TOPSIS

... TOPSIS
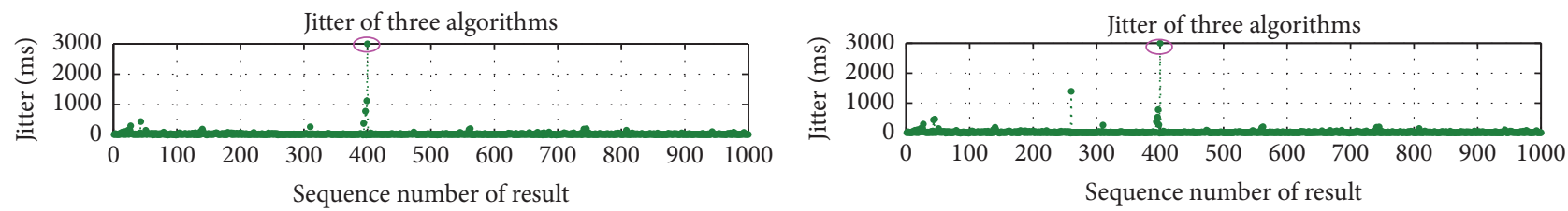

... FMADM

(a) Jitter of file service

(b) Jitter of video service

FIGURE 9: The jitter comparison of three algorithms. 

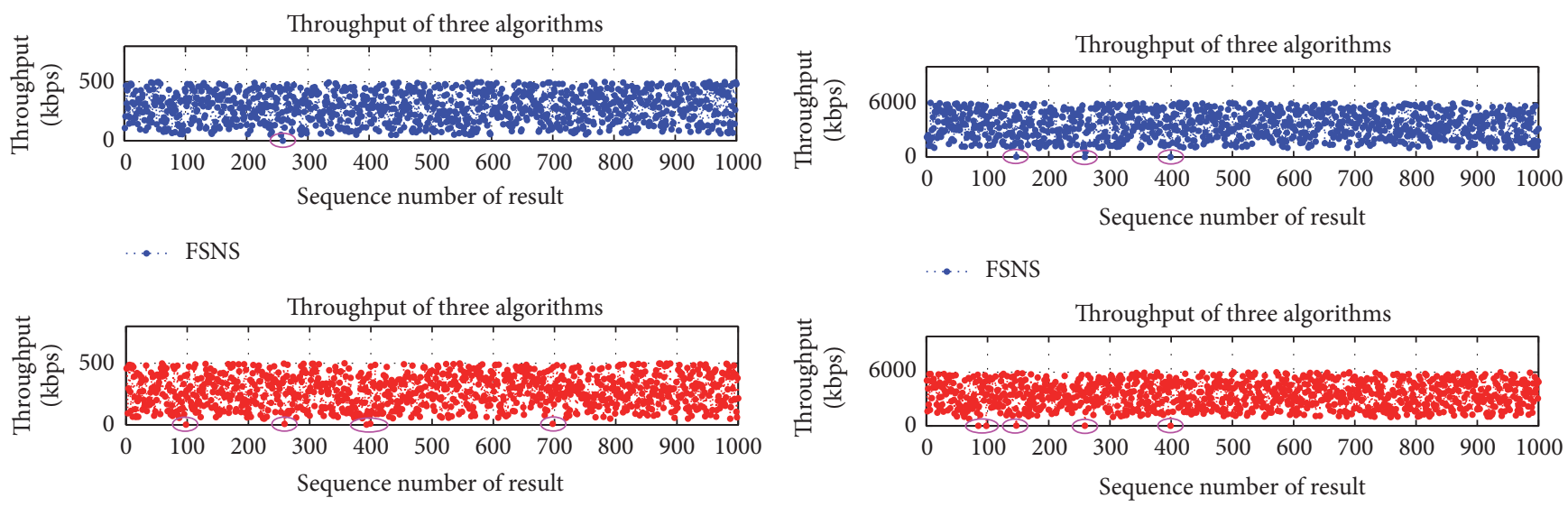

... TOPSIS

... TOPSIS

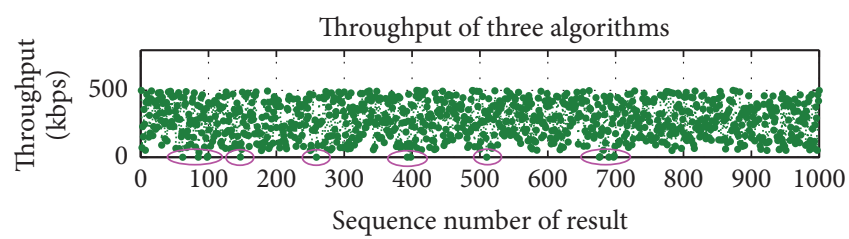

... FMADM

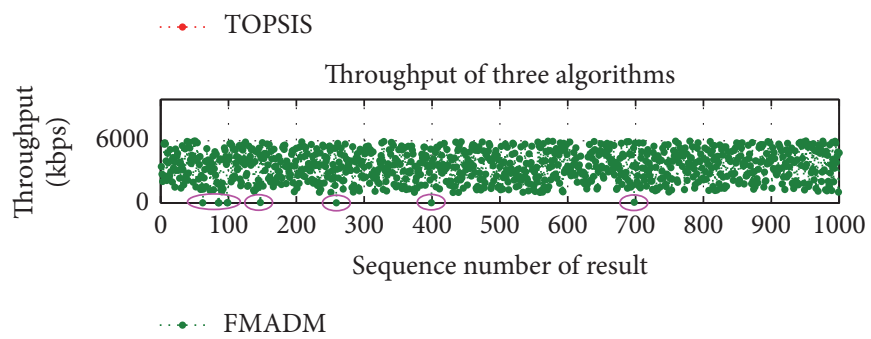

(a) Throughput of file service

(b) Throughput of video service

FIGURE 10: The throughput comparison of three algorithms.

to $100 \%$ which are marked by pink circles and is reduced by using FSNS. The numbers of file service are 2, 5, and 9 by using FSNS, TOPSIS, and FMADM, respectively. The numbers of video service are 3, 5, and 7, correspondingly. (3) FSNS improves the performance of packet loss ratio metric from the point of overall differences compared with FMADM.

Thirdly, Figure 9 shows the jitter performance of FSNS outperforms TOPSIS and FMADM in the following aspects: (1) FSNS decreases the overall jitter compared with TOPSIS. FSNS has $10.4 \%$ of results up to $50 \mathrm{~ms}$, in comparison with 16.8\% using TOPSIS and 9.0\% using FMADM. (2) FSNS reduces the number of extreme jitters compared with FMADM. We observe that the 399th results of jitter are the extreme value in Figures 9(a) and 9(b). It shows that FSNS can better avoid the extreme jitter compared with FMADM in spite of the inconspicuous improvement.

Fourthly, Figure 10 shows that the throughput performance of FSNS has an advantage over TOPSIS and FMADM. The number of results reached to $0 \mathrm{kbps}$ which are marked by pink circles and is reduced by using FSNS. The numbers of file service are 1, 5, and 11 by using FSNS, TOPSIS, and FMADM, respectively. The numbers of video service are 3, 5, and 7 , correspondingly.

5.2.3. Mean and Standard Deviation Comparison. We further analyze the stability of FSNS, TOPSIS, and FMADM by the statistical analysis of their simulation results. The means and standard deviations of network delay, jitter, and packet loss ratio are shown in Figures 11-12. The average values of three algorithms calculated by two services represent the improvement trend. In Figure 11(a), the mean values of delay for three algorithms (i.e., FSNS, TOPSIS, and FMADM) are $125.13,136.31,255.08$ for file service, respectively. They are $122.29,125.64,238.34$ for video service, respectively. In Figure 12(a), the standard deviation values of delay for these three algorithms are 215.18, 242.60, 391.64 for file service, respectively. They are 243.34, 270.63, 377.86 for video service, respectively. We can observe that FSNS is only slightly better than TOPSIS but it is remarkably better than FMADM from the point of delay performance. FSNS has an average $5.5 \%$ decrement of mean and $10.7 \%$ decrement of standard deviation compared with TOPSIS. FSNS has an average $49.9 \%$ decrement of mean and $40.4 \%$ decrement of standard deviation compared with TOPSIS. In Figure 11(b), the mean values of delay for three algorithms are $0.57,0.82,2.52$ for file service, respectively. They are $0.67,0.75,2.05$ for video service, respectively. In Figure 12(b), the standard deviation values of delay for these three algorithms are 4.69, 7.11, 11.40 for file service, respectively. They are 5.88, 7.11, 9.87 for video service, respectively. In Figure 11(c), the mean values of delay for three algorithms are 25.13, 28.89, 32.57 for file service, respectively. They are $30.30,32.20,35.02$ for video service, respectively. In Figure 12(c), the standard deviation values of delay for these three algorithms are 19.38, 22.18, 107.03 for file service, respectively. They are 41.65, 44.18, 112.31 for video service, respectively. We can observe that FSNS is better than both TOPSIS and FMADM from the point of jitter performance. FSNS has an average 9.1\% decrement of mean and $8.0 \%$ decrement of standard deviation compared with TOPSIS. FSNS has an average $18.0 \%$ decrement of mean and $72.2 \%$ decrement of standard deviation compared with TOPSIS. We can observe that FSNS is only slightly 


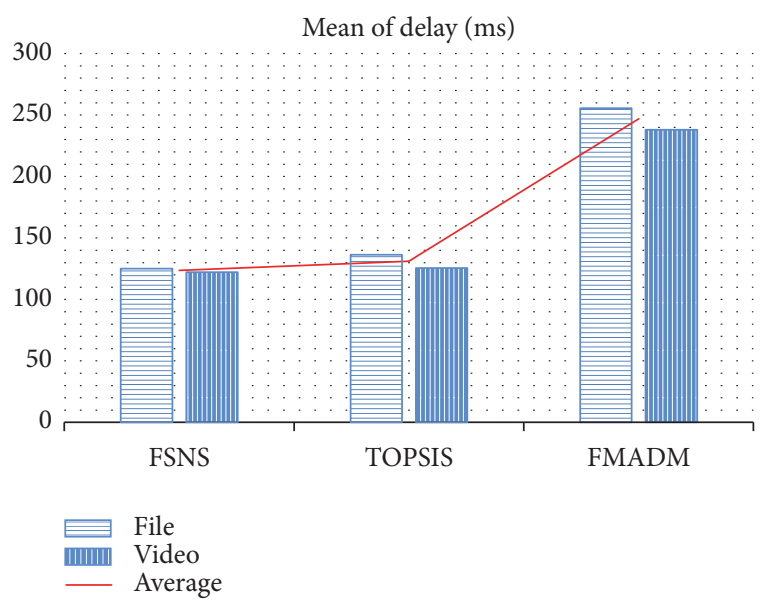

(a) Mean comparison of delay

Mean of packet loss ratio (\%)

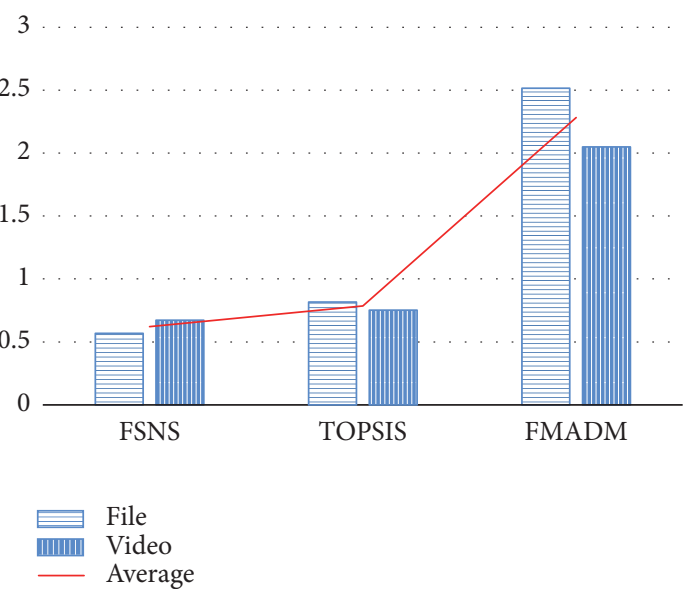

(b) Mean comparison of PLR

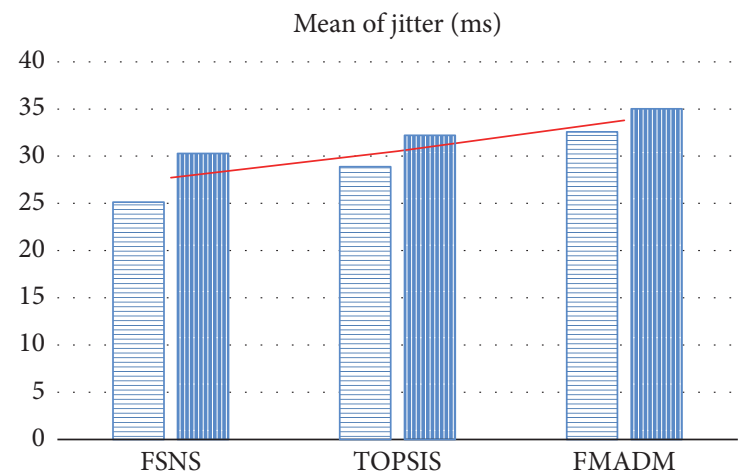

\begin{tabular}{ll}
\hline File \\
Video \\
Average
\end{tabular}

(c) Mean comparison of jitter

FIGURE 11: The mean comparison of performance metrics of three algorithms.

better than TOPSIS but it is remarkably better than FMADM from the point of delay performance. FSNS has an average

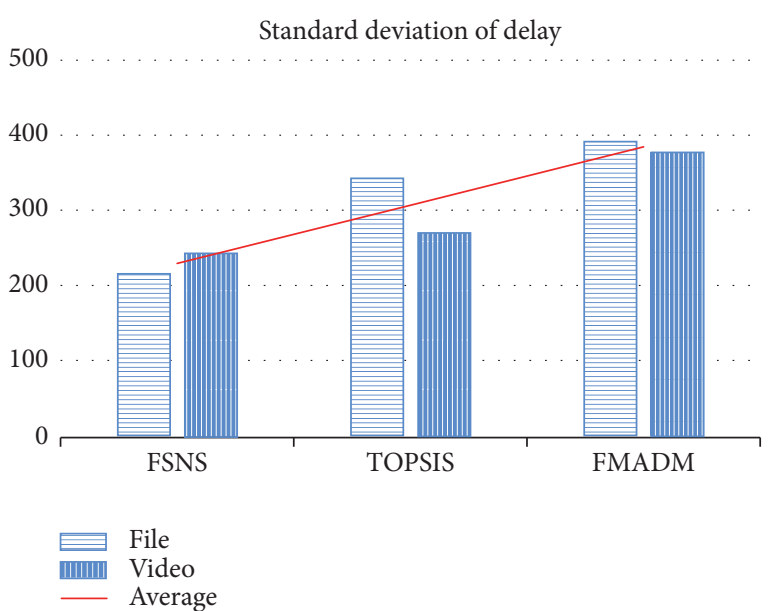

(a) Standard deviation comparison of delay Standard deviation of packet loss ratio

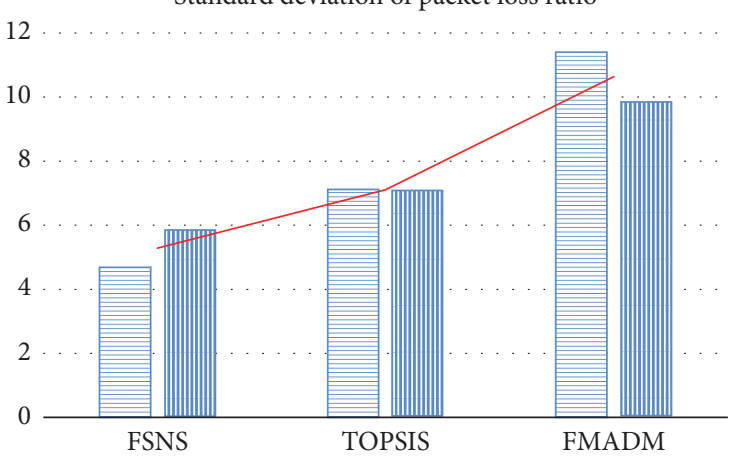

\begin{tabular}{ll}
\hline & File \\
\hline Video \\
Average
\end{tabular}

(b) Standard deviation comparison of PLR Standard deviation of jitter

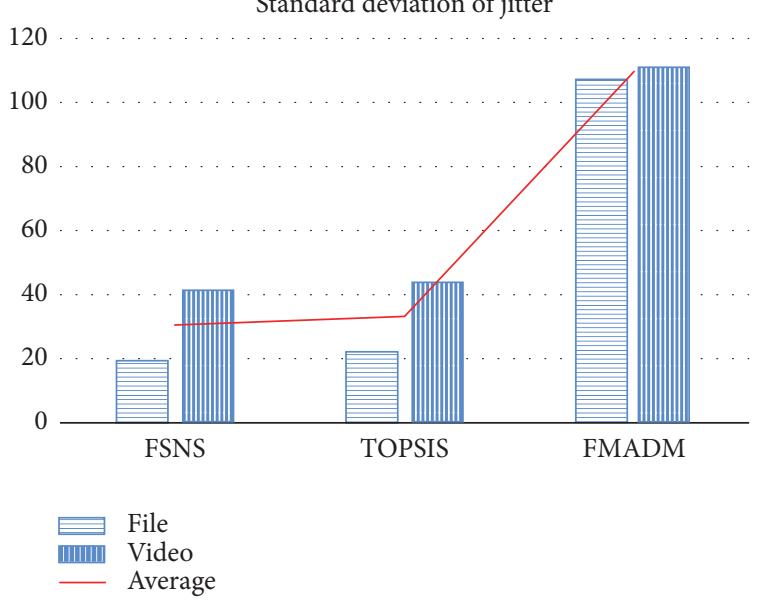

(c) Standard deviation comparison of jitter

FIGURE 12: The standard deviation comparison of performance metrics of three algorithms.

$21.0 \%$ decrement of mean and $25.7 \%$ decrement of standard deviation compared with TOPSIS. FSNS has an average $72.7 \%$ decrement of mean and $50.3 \%$ decrement of standard 
deviation compared with TOPSIS. All these results indicate that the performance ranking of three network selection algorithms is FSNS > TOPSIS > FMADM. Therefore, with relative low mean values and relative low standard deviation values of delay, jitter, and packet loss ratio, FSNS can be relatively stable to meet $Q$ oS requirements.

In conclusion, we find that FSNS is superior to both TOPSIS and FMADM in three ways no matter video or file service: (1) FSNS improves the network performance to some degree on the whole, mainly in terms of delay and packet loss ratio; (2) FSNS is better to avoid to select the poor wireless network; (3) FSNS can be relatively stable to meet QoS requirements.

\section{Conclusion}

In this paper, we first propose FSNS, a novel dynamic imprecise-aware network selection approach for the HSR scenario. The fuzzy logic is introduced to deal with the imprecise attributes for the network selection problem. Moreover, utility functions are presented for the calculation of overall satisfaction of multiple attributes to select the best network by integrating QoS requirements with actual network quality. We carry out plenty of simulations by using real-world data to validate the effectiveness and performance of FSNS. The results of the evaluation indicate that FSNS has a good performance improvement and a preferable decision compared with TOPSIS and FMADM for both video and file transmission services. The preferable decision of FSNS mechanism maintains relative stability and reduces abnormal selections. Since the data is collected from the real world, we think it has some extent pragmatic value. This paper has alleviated the abnormal network selection due to the imprecision and mobility problems in high-speed railway scenarios. The load balancing problem is also an important issue in this field. For future work, we plan to consider a dynamic adaptive load balancing mechanism based on our actual measurement environment.

\section{Abbreviations}

$s_{j}: \quad j$ th ID of service types

$\mathrm{CN}_{i}$ : $i$ th ID of candidate networks

$w f_{j}^{k}$ : Fuzzy weight of attribute $k$ for service $s_{j}$

$w c_{j}^{k}$ : Defuzzified weight of attribute $k$ for service $s_{j}$

$w_{j}^{k}$ : Normalized weight of attribute $k$ for service $s_{j}$

$u_{j}^{k}$ : QoS upper bound of attribute $k$ for service $s_{j}$

$l_{j}^{k}$ : QoS lower bound of attribute $k$ for service $s_{j}$

$\theta_{j}^{k}$ : QoS threshold of attribute $k$ for service $s_{j}$

$x_{i}^{k}$ : Value of attribute $k$ in network $\mathrm{CN}_{i}$

$x f_{i}^{k}$ : Fuzzified value of attribute $k$ in network $\mathrm{CN}_{i}$

$\mathrm{Sat}_{i j}^{k}$ : Satisfaction of network $\mathrm{CN}_{i}$ to service $s_{j}$

$\mathrm{Sd}_{i j}$ : Overall satisfaction of network $\mathrm{CN}_{i}$ to service $s_{j}$.

\section{Conflicts of Interest}

The authors declare that there are no conflicts of interest regarding the publication of this paper.

\section{Acknowledgments}

This work is supported by the Fundamental Research Funds for the Central Universities under Grant nos. 2017YJS028, 2017JBM018, and 2016JBM004, the Electronic Information Technology Innovation and Cultivation under Grant no. Z171100001217004, and the National Natural Science Foundation of China under Grant no. 61232017.

\section{References}

[1] D. Niyato and E. Hossain, "Dynamics of network selection in heterogeneous wireless networks: an evolutionary game approach," IEEE Transactions on Vehicular Technology, vol. 58, no. 4, pp. 2008-2017, 2009.

[2] W. Guo, S. Wang, X. Chu, J. Zhang, J. Chen, and H. Song, "Automated small-cell deployment for heterogeneous cellular networks," IEEE Communications Magazine, vol. 51, no. 5, pp. 4653, 2013.

[3] S. Sagari, I. Seskar, and D. Raychaudhuri, "Modeling the coexistence of LTE and WiFi heterogeneous networks in dense deployment scenarios," in Proceedings of the IEEE International Conference on Communication Workshop, ICCW 2015, pp. 23012306, London, UK, June 2015.

[4] J. Haydar, A. Ibrahim, A. E. Samhat, and G. Pujolle, "ABCDecision: A simulation platform for access selection algorithms in heterogeneous wireless networks," EURASIP Journal on Wireless Communications and Networking, vol. 2010, Article ID 787098, 2010.

[5] L. Wang and G. S. G. S. Kuo, "Mathematical modeling for network selection in heterogeneous wireless networks - A Tutorial," IEEE Communications Surveys \& Tutorials, vol. 15, no. 1, pp. 271292, 2013.

[6] B. Ai, X. Cheng, T. Kurner et al., "Challenges toward wireless communications for high-speed railway," IEEE Transactions on Intelligent Transportation Systems, vol. 15, no. 5, pp. 2143-2158, 2014.

[7] G. H. Tzeng and J. J. Huang, "Multiple attribute decision making," Lecture Notes in Economics and Mathematical Systems, vol. 375, no. 4, pp. 1-531, 2011.

[8] R. Trestian, O. Ormond, and G.-M. Muntean, "Game theorybased network selection: solutions and challenges," IEEE Communications Surveys \& Tutorials, vol. 14, no. 4, pp. 1212-1231, 2012.

[9] B. Korte and J. Vygen, Combinatorial Optimization:Theory and Algorithms, Springer, 2000.

[10] E. Altman, "Applications of Markov Decision Processes in Communication Networks," in Handbook of Markov Decision Processes, vol. 40 of International Series in Operations Research \& Management Science, pp. 489-536, Springer, Boston, Mass, USA, 2002

[11] E. V. D. Berg, P. Gopalakrishnan, B. Kim et al., "Dynamic network selection using kernels," in Proceedings of the 2007 IEEE International Conference on Communications, ICC'07, pp. 6049-6054, Glasgow, Scotland, June 2007.

[12] S. Kher, A. Somani, and R. Gupta, "Network selection using fuzzy logic," in Proceedings of the 2nd International Conference on Broadband Networks, 2005, pp. 941-950, Boston, Mass, USA.

[13] A. N. Kolmogorov, Foundations of the Theory of Probability, Chelsea Publishing Company, New York, NY, USA, 1950. 
[14] Q. Xiao, K. Xu, D. Wang, L. Li, and Y. Zhong, "TCP Performance over Mobile Networks in High-Speed Mobility Scenarios," in Proceedings of the 2014 IEEE 22nd International Conference on Network Protocols (ICNP), pp. 281-286, October 2014.

[15] L. Li, K. Xu, D. Wang, C. Peng, Q. Xiao, and R. Mijumbi, “A measurement study on TCP behaviors in HSPA+ networks on high-speed rails," in Proceedings of the 34th IEEE Annual Conference on Computer Communications and Networks, IEEE INFOCOM 2015, pp. 2731-2739, Hong Kong, China, May 2015.

[16] E. Stevens-Navarro, Y. Lin, and V. W. S. Wong, "An MDP-based vertical handoff decision algorithm for heterogeneous wireless networks," IEEE Transactions on Vehicular Technology, vol. 57, no. 2, pp. 1243-1254, 2008.

[17] M. A. Senouci, M. S. Mushtaq, S. Hoceini, and A. Mellouk, "TOPSIS-based dynamic approach for mobile network interface selection," Computer Networks, vol. 107, pp. 304-314, 2015.

[18] T. Bouali and S. Senouci, "A fuzzy logic-based communication medium selection for QoS preservation in vehicular networks," in Proceedings of the ICC 2016 - 2016 IEEE International Conference on Communications, pp. 1-6, Kuala Lumpur, Malaysia, May 2016.

[19] M. A. Senouci, M. R. Senouci, S. Hoceini, and A. Mellouk, "An evidential approach for network interface selection in heterogeneous wireless networks," in Proceedings of the 59th IEEE Global Communications Conference, GLOBECOM 2016, Washington, Wash, USA, December 2016.

[20] W. Chen, S. Gong, and X. Jiang, "Fuzzy multiple attribute decision access scheme in heterogeneous wireless network," Multimedia Tools and Applications, pp. 1-17, 2017.

[21] F. Dernoncourt, Introduction to fuzzy logic, Institute of Technology, 2013.

[22] S. J. Chen and C. L. Hwang, Fuzzy Multiple Attribute Decision Making, Springer, Berlin, Germany, 1992.

[23] P. C. Fishburn, "Utility theory for decision making," Publications in Operations Research, vol. 22, pp. 308-309, 1970.

[24] S. Mammen, Making sense of sinal strength/signal quality readings for cellular modems, http://blog.industrialnetworking .com/2014/04/making-sense-of-signal-strengthsignal.htm.

[25] N. Seitz, "ITU-T QoS standards for IP-based networks," IEEE Communications Magazine, vol. 41, no. 6, pp. 82-89, 2003.

[26] X. Wang, D. Qu, K. Li et al., "A flexible and generalized framework for access network selection in heterogeneous wireless networks," Pervasive and Mobile Computing, vol. 40, pp. 556576, 2017.

[27] R. He, B. Ai, G. Wang et al., "High-Speed railway communications: from GSM-R to LTE-R," IEEE Vehicular Technology Magazine, vol. 11, no. 3, pp. 49-58, 2016.

[28] Ubuntu, http://www.ubuntu.org.cn/global.

[29] P. Dong, B. Song, H. Zhang, and X. Du, "Improving Onboard Internet Services for High-Speed Vehicles by Multipath Transmission in Heterogeneous Wireless Networks," IEEE Transactions on Vehicular Technology, vol. 65, no. 12, pp. 9493-9507, 2016. 


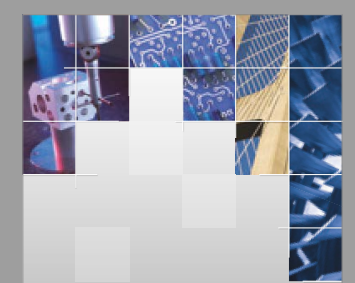

\section{Enfincering}
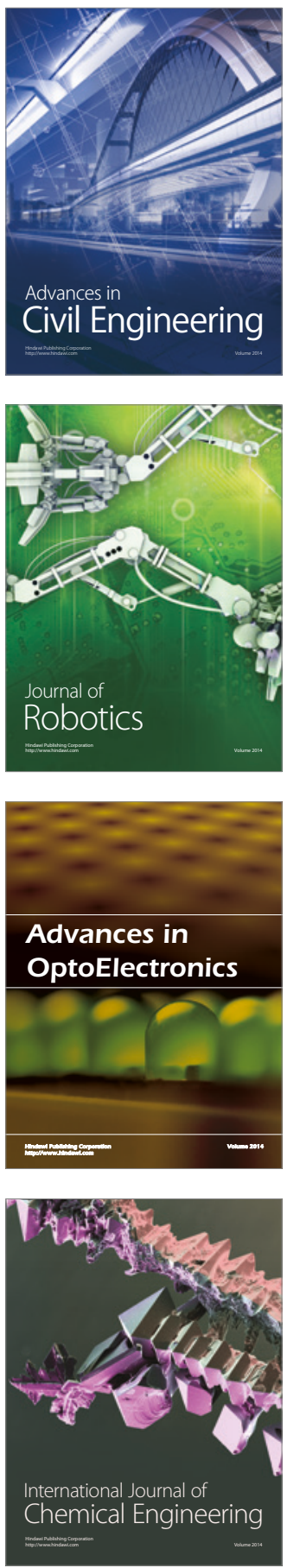

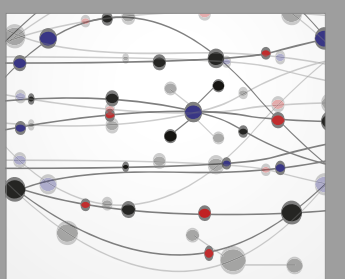

The Scientific World Journal

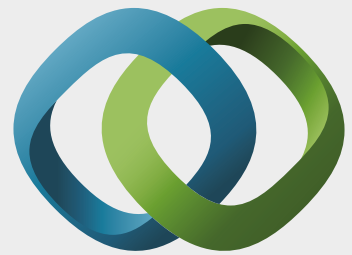

\section{Hindawi}

Submit your manuscripts at

https://www.hindawi.com
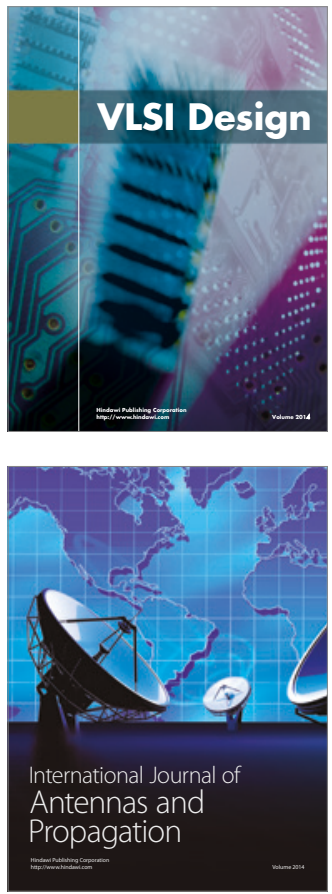

\section{Rotating}

Machinery
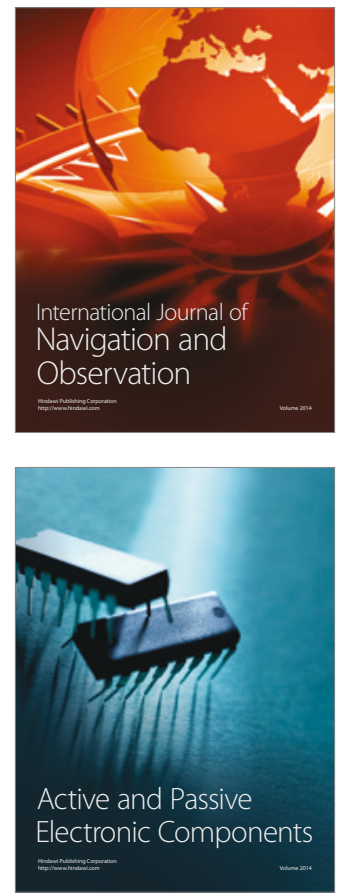
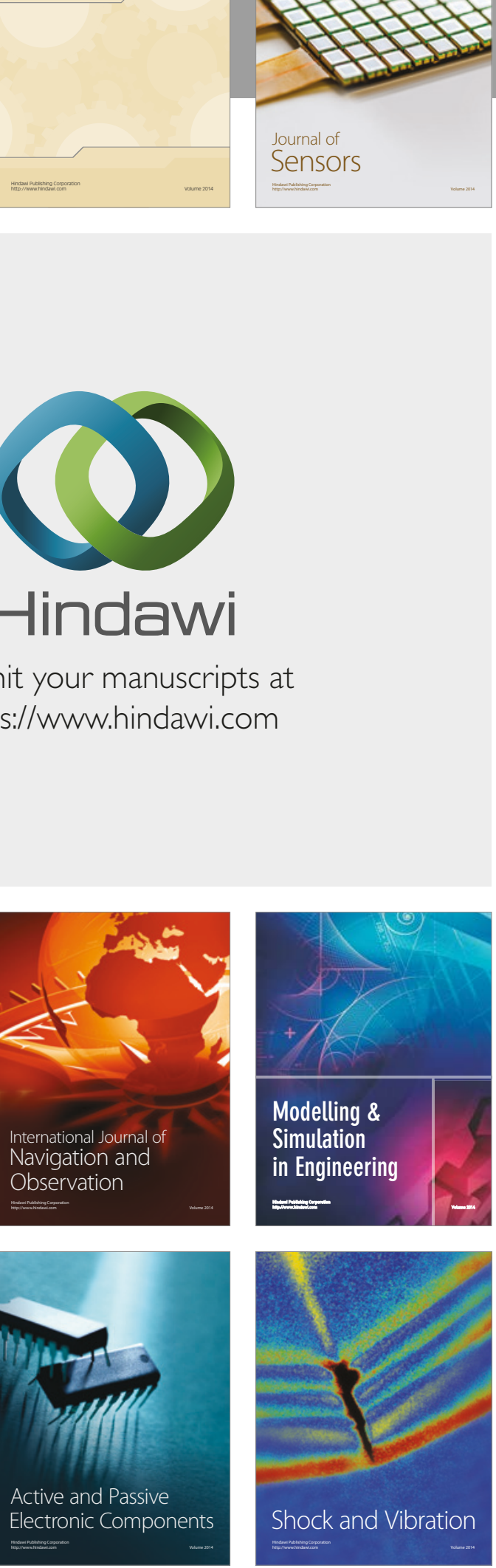
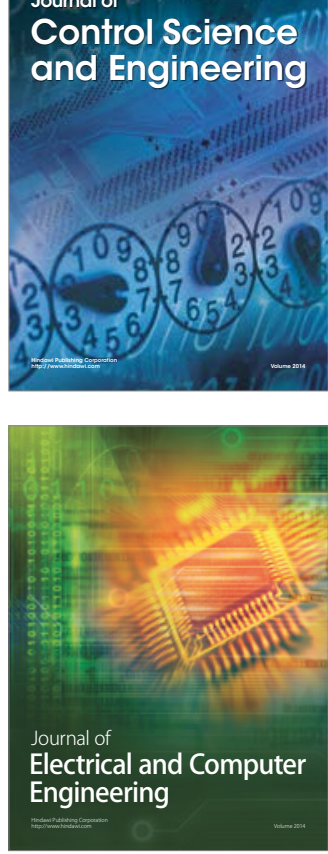

Distributed

Journal of

Control Science

and Engineering
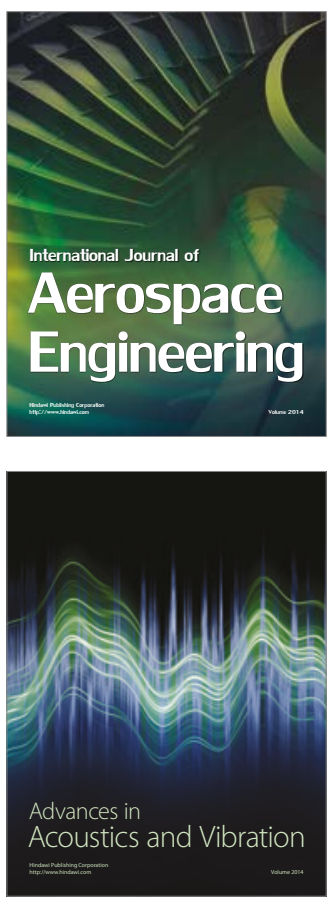

Sensor Networks 